\title{
Las primeras adecuaciones litúrgicas en el fondo de la Comisión Central Pontificia de Arte Sacro en Italia
}

\author{
The First Liturgical Adjustments in the Archive of the Central Pontifical Commission for Sacred Art in Italy
}

Barbara Fiorini · Investigadora independiente, Roma (Italia), fioriniarchitettura@gmail.com

Recibido: $17 / 09 / 2019$

Aceptado: 29/11/2019

(c) BY-NC https://doi.org/10.17979/aarc.2019.6.0.6221

\section{RESUMEN}

Desde 1924, operaba en Italia la Comisión Central Pontificia de Arte Sacro en Italia, un ente de la Santa Sede creado en 1924 por Pío XI como órgano asesor y ejecutivo, con el objetivo de proteger y promover el arte sacro. En el transcurso de unos 60 años de actividad, llegaron a la Comisión Central Pontificia más de 6.000 proyectos para su examen.

Durante el Concilio Vaticano, el presidente de esta Comisión Pontificia fue Giovanni Fallani, compilador del capítulo sobre el arte en la liturgia de la constitución Sacrosanctum Concilium.

Desde la desclasificación del fondo, recientemente inventariado, del Archivo Secreto Vaticano, es posible reconstruir un episodio inédito sobre cómo se trató en Italia la adaptación de las iglesias después del Concilio por parte de los que habían participado operativamente en él. Y por lo tanto, es posible evaluar la correspondencia de los proyectos a los preceptos de la liturgia y del arte sacro.

\section{PALABRAS CLAVE}

Concilio Vaticano II, Giovanni Fallani, arquitectura religiosa, Comisión Central Pontificia de Arte Sacro

\section{ABSTRACT}

Since 1924, The Central Papal Commission for Religious Works of Art in Italy, an organ of The Holy See, with advisory and executive functions, has been operating with the aim of protecting and promoting sacred art. More than 6000 projects have been submitted to the Commission over about 60 years.

Giovanni Fallani was the president of this Papal Commission during the Second Vatican Council. He is the drafter of the chapter included in the document Sacrosantum Concilium, concerning the art into the liturgy.

From the fund counting, recently inventoried in the Vatican Secret Archive, we can reconstruct an unpublished cross-section regarding the ways of churches adaptation in the aftermath of the Council. In detail our researches focused on the compliance level of the projects with the liturgy and sacred art precepts by those who had actively participated into the provisions drafting.

\section{KEYWORDS}

Vatican II, Giovanni Fallani, Sacred Architecture, The Central Papal Commission for Religious Works of Art

Cómo CITAR: Fiorini, Barbara. 2019. «Las primeras adecuaciones litúrgicas en el fondo de la Comisión Central Pontificia de Arte Sacro en Italia». Actas de Arquitectura Religiosa Contemporánea 6: 42-49. https://doi.org/10.17979/aarc.2019.6.0.6221. 


\section{INTRODUCCIÓN}

El objetivo de esta comunicación es el de arrojar luz sobre el debate inherente a la reforma litúrgica que acompañó los años del Concilio Vaticano II y los que inmediatamente le siguieron, a través del examen de documentos de archivo recientemente inventariados en el Archivo Secreto Vaticano (ASV).

Hoy, cincuenta años después, interpretar las normas conciliares y aproximarse al tema de la adecuación litúrgica todavía es muy complicado. Revisando el fondo que se conserva en el archivo vaticano es posible reconstruir una visión transversal sobre cómo se trató en Italia el tema de la liturgia, el arte y la arquitectura por parte de aquéllos que habían participado operativamente en el Concilio y se encontraban evaluando la respuesta de los proyectos a los preceptos de la liturgia y del arte sacro.

El fondo al que me refiero es el de la Pontificia Commissione Centrale per l'Arte Sacra en Italia (PCCASI), ente de la Santa Sede instituido el 1 de septiembre de 1924 por voluntad de Pío XI, como órgano consultivo y ejecutivo orientado a la tutela $\mathrm{y}$ a la promoción del arte sacro en Italia. La Comisión Pontificia, en concreto, evaluaba y aprobaba los proyectos según los preceptos litúrgicos y del arte sacro, que después eran examinados, en su parte administrativo económica, por la administración estatal, y contó entre sus miembros y consultores con personajes de primera fila en la arquitectura italiana.

\section{GIOVANNI FALLANI Y EL CONCILIO}

El 17 de mayo de 1956 moría Giovanni Costantini, que había presidido la Comisión desde 1943, y le sucedía Giovanni Fallani, vicepresidente desde 1947.

Fallani participó en la tercera y en la cuarta sesión del Concilio Vaticano II. Desde 1953 hasta 1967 dirigió la revista Fede e Arte, en la cual, en 1962 publicó un artículo con el título "In preparazione al Concilio Vaticano II. L'arte Sacra e la liturgia", en el que evidenciaba el resultado del trabajo de más de cincuenta miembros de la Comisión Pontificia De Sacra Liturgia, listo para remitir a los padres conciliares. ${ }^{1}$ En el artículo, Fallani recordaba la carta pastoral del arzobispo de Milán, Giovanni Battista Montini, futuro papa Pablo VI, a la archidiócesis ambrosiana en la cuaresma de 1958, en la cual el cardenal evidenciaba la necesidad de potenciar la vida religiosa mediante el renacimiento litúrgico, e indicaba cómo infundir en la asamblea el sentido de una acción común. Montini recordaba que para participar en la liturgia era necesario ver, escuchar y comprender, y que participar quería decir actuar (Montini 1962).

Los conceptos básicos estaban muy claros, los movimientos litúrgicos de más allá de los Alpes y algunas experiencias italianas habían hecho escuela, pero todavía existía mucha confusión sobre las aplicaciones prácticas.

El mismo Montini, un año antes, había escrito a Fallani para obtener una interpretación informal del decreto de la Sagrada Congregación de Ritos relativo a la colocación del tabernáculo Sanctissimam Eucharistiam (01/04/1957).

Crea alguna dificultad la aplicación, o interpretación, del número cuatro: 'In ecclesiis, ubi unicum extat altare, hoc nequit aedificari, ut sacerdos celebret populus versus; sed super ipsum altare, in medio, poni debet tabernaculum'. Ahora: $¿$ Se trata sólo de los altares nuevos a edificar (aedificari) o también de los altares ya edificados? Si el altar cara al pueblo sostiene de forma digna el tabernáculo, ¿está igualmente proscrito? (...) ¿Es posible un altar doble, uno abajo y otro en alto, en forma de mesa, vuelto hacia el pueblo, para las funciones que reclaman un mayor contacto del sacerdote con el pueblo; el otro, siempre sobre el eje central de la iglesia, más bajo, vuelto hacia el ábside, con el tabernáculo dignamente colocado, para la celebración ordinaria de la Santa Misa, para la conservación y para la distribución de la Santísima Eucaristía? Esta parece la mejor solución; se necesitaría describirla bien, de modo que aparezca clara la observancia de la norma litúrgica relativa al tabernáculo, y sea al mismo tiempo posible la celebración de la Santa Misa del sacerdote con el rostro vuelto hacia el pueblo, circunstancia ésta que se demuestra siempre útil y deseable, si se quiere dar a la participación litúrgica un desarrollo fácil y ordenado. Resultaría, con esta solución, un complejo de dos altares: uno más alto, sin apenas desarrollo arquitectónico, y capaz de encuadrar decorosamente el otro altar inferior, con el tabernáculo que no emerge - o emerge poco- sobre la mesa del altar superior, mientras es normalmente colocado sobre 
el altar inferior. Tenemos ya en algunas capillas esta colocación bifronte, y da un resultado verdaderamente feliz; es indicado, principalmente, en las capillas de Comunidad, donde la celebración de la Misa con el sacerdote vuelto hacia el pueblo es más fácil (...) Pero en las iglesias que tienen vida pastoral popular las ventajas son evidentes: durante la semana, la Santa Misa se celebra en el altar inferior, donde está el tabernáculo y donde se dispensa la comunión a los fieles, mientras que la Misa festiva (...) se celebra en el altar superior, con el sacerdote realizando un coloquio litúrgico con la asamblea de los fieles (ASV/PCCASI/AG 150/15).

El 30 de noviembre de 1957, Fallani respondía que la norma parecía referirse tanto a los altares nuevos como a los ya edificados, y que el altar mirando al pueblo con un tabernáculo digno encima no estaba proscrito. La solución del altar dúplice no le parecía opuesto al decreto, ya que cada uno de los dos altares habría tenido una función muy precisa. Proponía además que se estudiara a opción de disponer un altar único sobre el presbiterio con un tabernáculo en el centro, en parte encajado en la 'mensa', para no impedir la vista del celebrante. Concluía asegurando a Montini que encargaría personalmente a algunos consultores de la PCCASI el estudio de esta importante cuestión, sometiendo luego a los liturgistas los eventuales ejemplos.

Fallani, en 1962, había redactado el capítulo séptimo del documento del Concilio sobre la liturgia (Sacrosanctum Concilium), y Crispino Valenziano nos narra que cuando se enviaba un texto al aula conciliar para su discusión, se mandaba para ser 'martirizado': de hecho se llamaba 'texto mártir', es decir, un texto no demasiado desarrollado sobre el cual comenzar a discutir (Valenziano et al 1998, 57-58). La semana en la cual el texto de Fallani debía ser desbrozado, se denomina la semana negra del Concilio. El cardenal Ottaviani, Secretario de la Congregación del Santo Oficio, se había retirado del Concilio porque no compartía algunas líneas demasiado progresistas. Este hecho preveía la intervención del Papa con debates y explicaciones. Justo en aquella semana el texto fue aprobado sin mayores discusiones. Desde aquel momento monseñor Fallani - refiere Valenziano - intentó de todas las maneras suplir las carencias del texto aprobado incorporando directamente ejemplos prácticos. En 1969, escribió Orientamenti dell'arte sacra dopo il Vaticano II, un texto en el cual se inspiraron muchos proyectistas dedicados a la adaptación de las iglesias y que, como veremos, era recomendado en diversas diócesis como texto de referencia.

El cardenal Montini fue elegido papa en 1963. El 8 de diciembre de 1965 concluyó el Concilio, pero de los documentos recuperados del Archivo Secreto Vaticano emerge cómo ya poco antes diversas diócesis se habían aventurado en la adaptación del presbiterio según la nueva liturgia, y veremos, por tanto, en los ejemplos que siguen, cómo se movían la Comisión Pontificia, los comitentes y los proyectistas en esta nueva era postconciliar.

\section{LAS PRIMERAS ADECUACIONES LITÚRGICAS}

En febrero del sesenta y cinco, el obispo de la diócesis de Cariati enviaba un proyecto firmado por el profesor Luciano Bartoli (pintor de Milán), para la sistematización del prebiterio de la iglesia parroquial de Cirò Superiore. Es un proyecto que revela muchas dudas (ASV/PCCASI/AG 68/5).

Bartoli proponía dos soluciones. La primera que preveía mantener el retablo del altar, retirar la mensa adelantándola, y dejar como muebles, hacia la balaustrada, a derecha e izquierda, la sede y el ambón- no le convencía, por la distancia del altar a la asamblea y el escaño postizo y no dominante. La segunda, más invasiva, preveía conservar el retablo, quitar el tabernáculo, remover el altar y la predela, y erigir 'un parapeto' para crear un ambón con la sede del celebrante debajo; en posición avanzada respecto al ambón pero retirado sobre la derecha, colocaba un altar en forma de mesa, con el antiguo frontal y [diversas] piezas recuperadas de la demolición de altares secundarios sin valor artístico. Finalmente, proponía una variante de la segunda solución con el escaño en posición central, y el ambón y el altar próximos a la balaustrada, uno a la derecha y otro a la izquierda. Era ésta la solución que más le convencía, porque creaba una mayor unidad entre el presbiterio y la asamblea y permitía comenzar a dar la comunión de pie. 
Es evidente la falta de un pensamiento orgánico; son muchas las nuevas exigencias litúrgicas que salen a la luz y a las cuales se intenta dar una respuesta, pero sin llegar a un proyecto arquitectónico definido. Y de hecho, la PCCASI se expresa con el siguiente juicio:

La solución propuesta modifica de manera no orgánica todo el ambiente preexistente, y por tanto, se requiere el estudio de una nueva sistematización, en la cual las nuevas exigencias litúrgicas se armonicen con el presbiterio existente (ASV/PCCASI/ AG 68/5).

El 11 de abril, fue la posición del tabernáculo en referencia al altar coram populo lo que suscitó dudas. Monseñor Enrico Villa, de la archidiócesis de Milán, pedía aclaraciones a Fallani sobre la disposición del tabernáculo sobre la mensa. Los cardenales y los obispos sufragáneos sostenían que era una buena solución, mientras que a muchos sacerdotes no les agradaba. Enviaba también un boceto sobre cómo proponía colocar el tabernáculo en el altar coram populo. $^{2}$

En este caso, Fallani respondía que la solución propuesta parecía contrastar con lo que había establecido el reciente documento del Consilium ad Exsequendam Costitutionem de Sacra Liturgia. De hecho, entre las soluciones no aprobadas estaba: «Tabernaculum intra altare stabiliter constitutum, sive in anteriore facie altaris appareat, sive non» (ASV/PCCASI/AG 150/15).

El Consilium era un organismo instituido por Pablo VI para implementar la constitución sobre Sagrada Liturgia, y estaba presidido por el cardenal Lercaro, que como ejemplo de su presidencia, quiso en 1965, adaptar litúrgicamente la cripta de la catedral de San Pedro en Bolonia. ${ }^{3}$

Junto a casos ya estudiados y conocidos como éste, el fondo que he examinado proporciona material archivístico relativo a cerca de veinte proyectos de adecuación: las problemáticas — por lo demás recurrentes - manifiestan las dificultades encontradas para la colocación del nuevo altar hacia el pueblo, respecto al existente. En mayo de 1965, el obispo de Aosta, Maturino Blanchet, solicitaba un consultor para tener una opinión autorizada acerca de la sistematización del altar mayor de su catedral. La Pontificia Comisión enviaba al arquitecto Giuseppe Zander, que redactó un interesante informe, en el cual reconocía que el altar tardobarroco colocado en el largo coro, próximo al ábside, no respondía al espíritu de la renovada liturgia, y por lo tanto, lo consideraba inútil bajo el punto de vista funcional. Zander refería que algunos canónigos habían querido desmontarlo y removerlo, colocándolo en otro sitio, para poner en su lugar los tubos del órgano, que él, con la ayuda de las nuevas tecnologías, sostenía que podían estar dispuestos a lo largo de las paredes.

Zander era completamente contrario a la eliminación del altar del siglo dieciocho, por consideraciones arquitectónicas; el coro, por otra parte, era muy profundo, y por lo tanto no revelaba problemas de espacio para la realización de un nuevo altar. Sobre la sucesión prospectiva a lo largo del mismo eje de la cátedra episcopal y del altar, proponía una «decoración caprichosa y variada, suspendida con cuerdas que desciendan de las bóvedas, que con curvas suaves, se armonizase con el altar posterior, dejando transparentar ampliamente la arquitectura sin ocultarla» (ASV/PCCASI/AG 30/6). La PCCASI, una vez examinado el informe efectuado en el lugar por el consultor Zander, aprobó el contenido en líneas generales.

Los mismos criterios los adoptó Zander en Chiavari, donde el obispo le encargó, en 1968, el proyecto de adaptación del presbiterio de la catedral. En este caso, prestó también una particular atención a la función de la balaustrada, que creía que todavía tenía una doble utilidad práctica: «Sirve como interrupción o 'rostra' frente al atril o atriles para la lectura de la Epístola y del Evangelio y, además, todavía puede usarse para arrodillarse, no pareciendo del todo universal el nuevo consenso de recibir la Sagrada Comunión de pie». También creía que la eliminación nunca sería aceptada por la Superintendencia de Monumentos. Sobre el nuevo altar sostenía que había dos posibilidades:

Simple arquitectura que no emule los fuertes valores plásticos y de claroscuro de la iglesia, o un nuevo altar que hable un lenguaje plástico expreso en términos modernos (ASV/PCCASI/AG 68/5). 

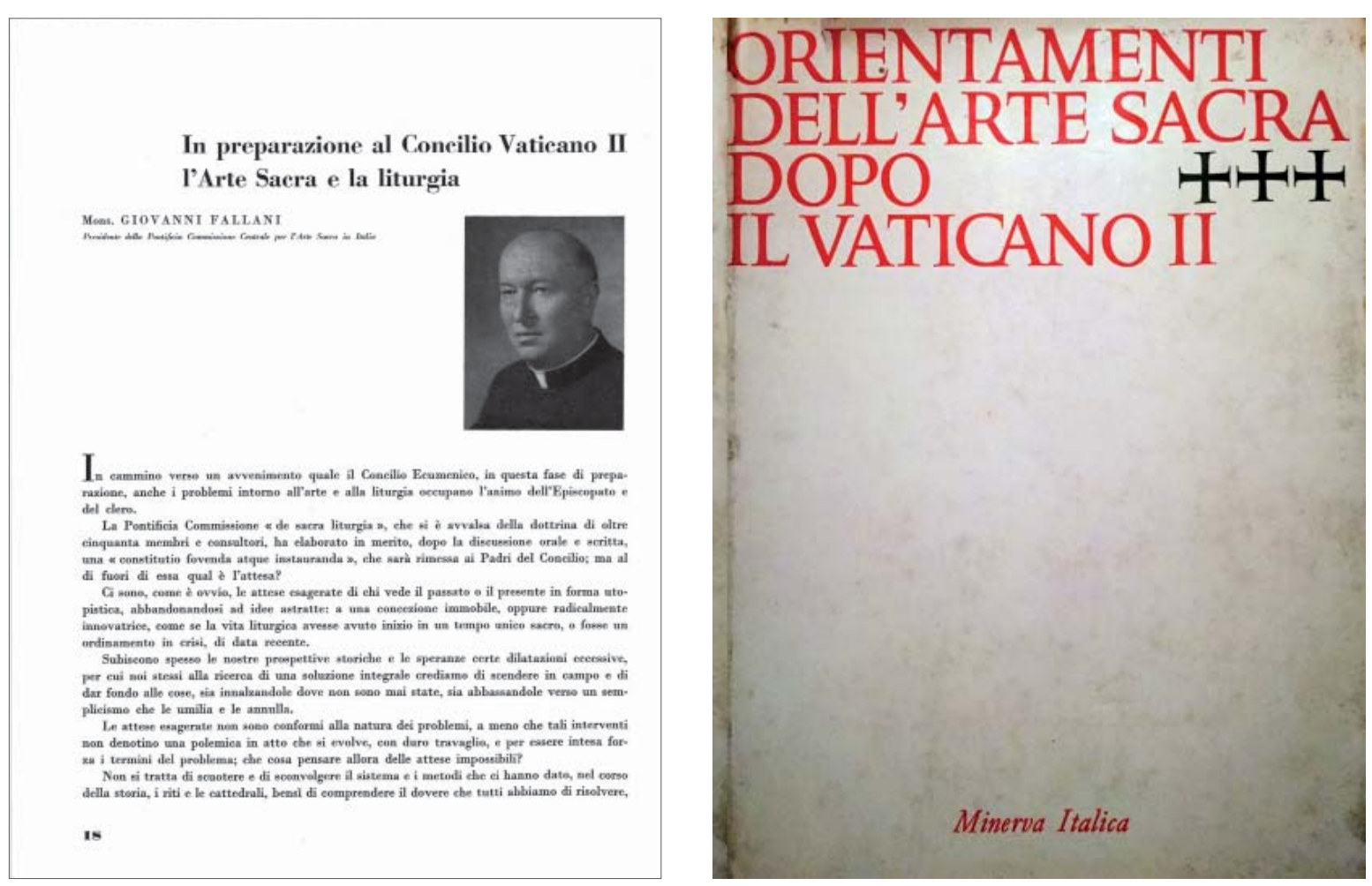

Fig. 01. Primera página del artículo de Giovanni Fallani «In preparazione al Concilio Vaticano II. L'Arte Sacra e la liturgia». Fede e Arte 1-2 (1962): 18-25.

Fig. 02. Portada del libro editado por Giovanni Fallani Orientamenti dell'arte sacra dopo il Vaticano II (1969).

Su opinión era la de descartar las imitaciones estilísticas, aunque la desnudez arquitectónica a menudo, para él, había conducido a ejemplos no lo bastante solemnes.

En febrero de 1970, la Comisión Diocesana de Arte Sacro de la curia episcopal de Oria, confesó en una carta enviada a la PCCASI, que encontraba no pocas dificultades en la sistematización del altar coram populo cuando el preexistente tenía un cierto valor artístico, en el número de los ambones, y en la posición del tabernáculo cuando no existiera una capilla lateral. Solicitaba indicaciones de libros o colecciones de ilustraciones (de soluciones ya adoptadas en otros sitios), útiles a este respecto (ASV/PCCASI/AG 180/4). Fallani respondió que la Comisión Pontificia ya había afrontado varias veces este tema en las últimas Semanas de Arte Sacro y en los congresos organizados en colaboración con la comisión episcopal de liturgia. Enviaba dos fascículos de la revista Fede e Arte que trataban de esa delicada cuestión y señalaba el reciente volumen preparado por él (AS ASV/PCCASI/AG 18/4). ${ }^{4}$

Entre los congresos, recuerdo el de Turín de 1967, con un interesante discurso inaugural del cardenal Pellegrino, en el cual, refiriéndose a las adaptaciones de la primera renovación postconciliar se lee:

Dejan perplejo por el resultado artístico y pastoral que han conseguido (...); arreglos postizos que no sólo son contrarios a las más elementales leyes del arte y del decoro, sino que no tienen ni siquiera el objetivo pastoral que la reforma se propone (Pellegrino 1967). 
El Consilium presidido por Lercaro se mantuvo activo hasta mil novecientos setenta (De Marchis 2013, 79-nota 298; Sanson 2008, 75-76). Todavía era demasiado pronto para que se alcanzara la consciencia de una innovación seguramente revolucionaria y compleja; las dudas derivadas de la falta de lineas-guía a las cuales atenerse y la poca difusión de las problemáticas, ampliaron todavía más la incertidumbre.

En 1974, la Conferencia Episcopal Italiana promulgaba las normas para la tutela y conservación del patrimonio histórico-artístico de la Iglesia. El apartado 13, 'Reforma litúrgica y nuevas adaptaciones', dice así:

La relación entre lo antiguo y lo nuevo será objeto de estudio, dentro del respeto de las recíprocas competencias, para obtener una mejor funcionalidad litúrgico-pastoral y una respuesta a las exigencias del arte (...) En toda adaptación, el altar debe conservar el carácter de centro ideal, en el cual converge la atención de la asamblea entera. Se debe prestar la máxima atención a la custodia eucarística, con una capilla digna y fácilmente accesible, o con la oportuna disposición del tabernáculo en el presbiterio. 5

En el mismo año, tras una solicitud para examinar el proyecto de la adaptación litúrgica de la catedral de Matelica, en la diócesis de Fabriano, la PCCASI, que había contribuido a la redacción del documento, respondía con indicaciones muy concretas.

En lo que se refiere a la sistematización del presbiterio, nada obsta al movimiento del altar y que pueda ser posteriormente desplazado unos $80 \mathrm{~cm}$. hacia la asamblea de los fieles. (...) Se aconseja la conservación en su sitio de la cátedra episcopal, que resultará mejorada en su visibilidad. Para la sede, parece oportuno adoptar el uso de muebles antiguos arrimándolos, preferiblemente, a la cara interna de las pilastras. Se aprueba la idea de utilizar como ambón la parte superior del púlpito lígneo (...), estudiándole un basamento en nogal, de valor arquitectónico, para elevarlo del plano del transepto (ASV/PCCASI/AG 101/11).

En 1977, tres años después de la redacción de las normas de tutela antes citadas, durante las obras de adecuación del presbiterio de la colegiata de Santa María Assunta en Camaiore, la Comisión Pontificia sostenía que la solución presentada, con el desmontaje del altar original y la reutilización de elementos para realizar uno nuevo, no era aceptable, y por lo tanto, sugería la conservación del retablo y la realización de un altar ex novo (ASV/PCCASI/ AG 101/63).

Los temas que se referían a la adaptación y el relativo acercamiento [entre el altar y los fieles] permanecían todavía inmutables, pero la Comisión ya comenzaba a expresarse de manera más concreta y clara, buscando una mediación entre conservación y renovación.

\section{DOS CASOS SINGULARES}

Entre todos los proyectos encontrados en el Fondo, uno sólo se sale del coro con una historia particular. Era en mayo de 1977 cuando el Vicariato de Roma informaba a Fallani que un grupo de feligreses de la iglesia de los Santos Mártires Canadienses en Roma, guiados por el coadjutor padre Guglielmo Amedei, había presentado una solicitud al Vicariato para una «reformulación del presbiterio de la iglesia parroquial». El tipo de propuesta adelantada, la falta de documentación suficiente y la oposición del proyectista de la iglesia - el profesor arquitecto Apollonj Ghetti- habían dejado al cardenal muy perplejo. Los mismos feligreses habían presentado, también, el proyecto a la PCCASI, y por lo tanto, el cardenal se dirigía a Fallani para que manifestase una opinión general, y él, antes de hacerlo, envió dos consultores para examinar la situación.

Ocurría que en enero de 1977, el arquitecto Mattia Del Prete y el párroco se habían reunido con el ingeniero Breccia (de la oficina técnica del Vicariato) y el padre Luca Brandolini (de la oficina litúrgica), explicándoles el proyecto y animándoles a experimentar. El proyecto preveía la realización de un diafragma al inicio del presbiterio, a la derecha del cual se debería disponer una capilla ferial, colocando el altar en el centro de la nave con la asamblea dispuesta en torno a él. Breccia y Brandolini afirmaron que para la nave se enfrentaban a un «discurso revolucionario», que no estaban preparados todavía para asumirlo, pero que podía ser al menos realiza- 
do a título experimental, con la convicción de que la aprobación definitiva llegaría, tras un tiempo de experimentación congruente, con el altar y el ambón definitivamente colocados.

Un mes después, la Comisión Pontificia rechazaba el proyecto considerándolo incompleto y carente de fundamentos litúrgicos, de concreción estilística, y de una adecuada representación gráfica. Consideraba necesario que — de acuerdo con Apollonj Ghetti- se elaborara «un proyecto detallado que comprendiese todas las estructuras y los objetos móviles e inmóviles, y esto también dada la presencia de obras de cualificados artistas modernos»(ASV/PCCASI/AG 101/168).Y manifestaba la duda de que la transformación del presbiterio en capilla ferial beneficiase la visión arquitectónica unitaria de la iglesia, y que no constituyese, por el contrario, una amputación arbitraria y de escaso resultado práctico, recordando que un experimento similar había estado funcionando en la cripta durante algún tiempo.

El 17 de septiembre, el mismo Fallani y su secretario Garlato se personaron en la iglesia para reunirse con Oscar Zanera, obispo auxiliar para el sector norte, con el padre Amadei, el arquitecto Apollonj Ghetti y un representante del padre general de los Sacramentinos, para encontrar una solución. En ese momento, Fallani preguntó el motivo por el que se había hecho un corte en la iglesia, creando una capilla más pequeña en el presbiterio y dos altares. El padre Amadei explicó que se habían querido seguir las indicaciones de la instrucción Eucharisticum Mysterium: un nuevo modo de celebrar la eucaristía y de hacer adoración, poniendo de relieve la íntima relación que existe entre ella y la celebración del memorial del Señor. Entonces Fallani le pidió al arquitecto Apollonj Ghetti que desarrollase un nuevo proyecto que satisfaciese tres necesidades: el altar lo más cerca posible de la gente, basado en una lógica constructiva; la creación de una conexión con el presbiterio a través de la sede del celebrante, que por lo tanto, no debería ser un elemento divisorio; la colocación del ambón en un lugar conveniente.

El 2 de agosto de 1978, la Comisión Pontificia recibió dos soluciones del arquitecto y aprobó una que respondía a lo solicitado. La experimentación se suspendió así, y los polos litúrgicos regresaron al presbiterio.

Este es un caso emblemático para explicar la complejidad del tema con respecto a la recepción de las indicaciones litúrgicas, los procedimientos y las formas en que los diversos actores abordaron el tema de la adaptación. Mientras que, por un lado, un grupo de feligreses solicitaba una renovación en nombre de una instrucción litúrgica, por otro, el arquitecto de la iglesia recibía la solidaridad de mil cien fieles indignados, firmantes de una petición en la que se expresaba la oposición al trabajo realizado, y la solicitud de que la iglesia recuperase el aspecto que tenía antes de su destrucción, señalando a los sacerdotes como «bárbaros innovadores» (ASV/PCCASI/AG $101 / 168)$.

El último caso examinado en orden cronológico es el proyecto de adaptación de la Catedral de la Natividad de la Virgen María, en Cassano Jonio, de 1980. Giovanni Carbonara, arquitecto experto en restauración, así como uno de los autores del texto editado por Fallani, tenía como objetivo conciliar las necesidades de adaptación funcional y litúrgica con las de conservación. Su propuesta se basaba en el concepto de reversibilidad y de una transparencia ideal y física mediante el uso del vidrio para la construcción de un nuevo altar, de modo que los muebles del siglo XVIII pudieran permanecer absolutamente intactos, visibles y en pleno uso.

En el informe que acompañaba al proyecto, Carbonara hablaba de una intervención que dejaba abierta la disponibilidad a posibles cambios futuros para futuras precisiones litúrgicas, para el progreso de la reflexión teológica sobre el arte sagrado o para impredecibles descubrimientos del área misma de la catedral. Todas las propuestas presentadas las refería a las indicaciones conciliares y, en particular, a las orientaciones que PCCASI había sugerido en casos similares a la hora de armonizar las necesidades de renovación con las de respeto por los valores históricos y artísticos de los monumentos (ASV/PCCASI/ AG 101/23). 


\section{CONCLUSIONES}

En conclusión, este examen muestra claramente el papel que desempeñaron la Comisión Pontificia y la figura de Fallani durante el período postconciliar. Dentro de un panorama de incertidumbre en el que clientes y profesionales intentaban navegar entre nuevas indicaciones litúrgicas, entre renovaciones y preexistencias, la comisión desempeñó el papel de coordinación, de un centro de debate, discusión y dirección; y sobre todo, fue capaz de divulgar y educar, poco a poco, en la aplicación de las normas conciliares.

\section{BIBLIOGRAFÍA}

1973. Notiziario della CEI, 2 (1 de marzo): 32-38.

ASV/PCCASI/AG. Arquivio Segreto Vaticano, Pontificia Commissione Centrale per l'Arte Sacra en Italia, Archivo General, \# carpeta, \# fascículo. Ciudad del Vaticano.

De Marchis, Daniele. 2013. L'archivio della Commissione Centrale per l'Arte Sacra in Italia, inventario. Ciudad del Vaticano: Archivio Segreto Vaticano.

Fallani, Giovanni, ed. 1969. Orientamenti dell'arte sacra dopo il Vaticano II. Bergamo: Minerva Italica.

Fallani, Giovanni. 1962. «In preparazione al Concilio Vaticano II. L'Arte Sacra e la iturgia». Fede e Arte 1-2: 18-25.

Gresleri, Giuliano. 2004. «Dopo il Concilio Vaticano II, Esperienze, speranze, sconfitte». In Chiesa e quartiere: storia di una rivista e di un movimento per l'architettura a Bologna, Giuliano Gresleri et al., 226-238. Bologna: Compositori.

Montini, Giovanni Battista. 1962. «Su l'educazione liturgica». Fede e Arte 3: 202-225.

Pellegrino, Michele. 1967. «Arte cultura e liturgia». Chiesa e Quartiere 42: 4-7.

Sagrada Congregación de Ritos. 1957. Decreto Sanctissimam Eucharistiam, 1 de abril. Acta Apostolicae Sedis 8: 425-426.

Sanson, Virginio. 2008. Architettura Sacra del Novecento. Padova: Messaggero.
Valenziano, Crispino, Gaspare Gruppuso, Leonardo Giordano. 1998. I nuovi luoghi liturgici nella cattedrale di Trapani. Trapani: Il pozzo di Giacobbe.

Villa, Enrico. 1965. Altare e Tabernacolo. Fede e Arte 4: 226-245.

\section{NOTAS}

1. El artículo formaba parte de las actas de la novena semana de arte sacro, que se celebró en Roma en 1961, y que fueron publicadas en el número 1-2 de la revista Fede e Arte de 1962 (Fallani 1962).

2. En un artículo publicado en 1965 en Fede e Arte, Villa, partiendo del decreto de la Sagrada Congregación de Ritos relativo a la colocación del tabernáculo, hace hace un examen sobre el altar y el tabernáculo.

3. Había sido instituido en 1963, y produjo muchas intervenciones en aplicación de la Sacrosanctum Concilium; entre ellos emerge la segunda Instructio Inter Oecumenici del 26 de septiembre de 1964, y las cartas del presidente del Consilium de 1965 y 1966, en las cuales se afirmaba la unicidad del altar en la celebración, y se consideraba poco aceptable su provisionalidad. Para profundizar en el tema puede verse Sanson 2008, 78.

4. El volumen al cual se hace referencia es Fallani 1969.

5. Un primer borrador de este documento se publicó, a petición del Consejo Permanente, en el Notiziario della CEI (1973), para su discusión en las Conferencias Regionales; las observaciones recibidas fueron enviados a la Comisión Pontificia para el Arte Sacro en Italia, que supervisó un segundo borrador, luego aprobado por la X Asamblea General (11-16/04/1973). El documento fue presentado a la autoridad superior para la aprobación necesaria. El 01/04/1974, mediante la carta $\mathrm{n}^{\circ}$ 256949, la Secretaría de Estado entregó el texto definitivo, con algunos retoques al borrador anterior sugeridos por la Embajada de Italia cerca de la Santa Sede.

\section{PROCEDENCIA DE LAS ILUSTRACIONES}

Archivo de la autora. 